\title{
Deep brain stimulation of Anteromedial Globus Pallidus internus improved OCD rather than tics in a Tourette's syndrome patient
}

\author{
Zahra Aminzade ${ }^{1}$, Sepand Tehrani Fateh ${ }^{1}$, Reza Jalili khoshnood ${ }^{2}$, Farzad Ashrafi ${ }^{2}$, and \\ Mehri Salari ${ }^{1}$ \\ ${ }^{1}$ Shahid Beheshti University of Medical Sciences \\ ${ }^{2}$ Shohada-e Tajrish Hospital
}

July 24, 2021

\begin{abstract}
After performing DBS surgery on a patient with Tourette syndrome, significant improvement was observed in OCD, but the tic remained unchanged, which contradicts previous studies. Herein we discuss that fluctuations in the therapeutic outcomes may be due to differences in physiological conditions tic- or OCD-specified pathways.
\end{abstract}

\section{Introduction:}

Tourette's syndrome (TS) is a chronic developmental neuropsychiatric disorder with an onset in early childhood, affected by both genetic and environmental factors. TS is characterized by multiple complex motor and vocal tics, in addition to some other psychological disorder such as attention deficit hyperactivity disorder (ADHD) and obsessive-compulsive disorder (OCD). The exact pathophysiology of TS is unclear but recent studies suggest that there may be a defect in the Cortico-Striato-Thalamo-Cortical (CSTC) circuit. Besides self-limited cases, some of TS patients with mild symptoms may need conventional treatments such as psycho-behavioral therapy, educational therapy and pharmacotherapy while refractory cases are potential candidates for deep brain stimulation (DBS) procedure which leads to an improvement in both tic component and other comorbidities of TS, but with different extents [1]. Herein we report a patient with TS which his OCD improved after DBS without his tic to be improved.

\section{Case presentation:}

A 32-year-old right handed gentleman was diagnosed with TS at the age of 5 with a progression through adolescence, presented by fluctuating complex motor tics including shoulder elevation, repetitive movement in the thumbs and eye blinking, which was accompanied by severe disabling OCD, beginning at the age of 8, sleep disorder for 15 years, adult onset ADHD, and anxiety disorder during the course of illness. He reported social impairment during school and at work. He had several unsuccessful behavioral- and pharmaco- therapies (table 1) since he was diagnosed. He had positive family history of TS in his father accompanied by OCD and simple motor tic in his sister. Due to severe symptoms, lack of response to multiple drugs unsuccessful psycho-behavioral therapy and patient's functional impairment, he was known as refractory case of TS and became a candidate for DBS surgery.

A consent was obtained from the patient and DBS surgery carried out under local anesthesia. Targets were determined and calculated via Medtronic S8 planning station software and approved via an MRI and microelectrode recording. Electrodes (3389/ Medtronic) were administered thourgh the coronal suture site, parallel to the midline and implanted symmetrically on the anteromedial globus pallidus interna (GPi) (Right: $\mathrm{x}=15.22, \mathrm{y}=6.40, \mathrm{z}=-7.74$ and Left: $\mathrm{x}=-14.32, \mathrm{y}=7.29, \mathrm{z}=-9.37)$. Stimulation test results during 
surgery demonstrated proper response with no immediate side effects. Post-operative Computed tomography scan demonstrated the appropriate position of electrodes. No complications were observed after the surgery. An IPG (Active PC/Medtronic) become connected to the leads and implanted subcutaneously in right chest wall.

One month after surgery the IPG was turned on, during assessment, right side stimulation showed no side effects while at the left side dyspnea related to ventral electrodes and paresthesia and vertigo related to dorsal electrodes were detected. The parameters were set on, Amplitude: 1, Pulse width: 80, frequency: 85, Bipolar on the left $\left(3^{+}, 0^{-}\right)$and unipolar on the right $\left(\mathrm{Case}^{+}, 8^{-}\right)$.

During follow up sessions, patient's condition was fluctuating in response to alterations in stimulation parameters as it is shown in table 1 . At the optimum parameters which was obtained after 8 months, OCD decreased dramatically; on the other hand, however, tics frequency and intensity did not change significantly and ultimately worsen during time. Patient's improvement in OCD also caused his medications to be reduced (table 1).

\section{Discussion:}

Different studies have revealed approximate circuits of tics, OCD and also TS which are generally known as CSTC circuit [1-3]. CSTC components including thalamus, posterolateral GPi and anteromedial GPi are among the most used targets for DBS procedure in TS, while other targets including subthalamic nucleus (STN), globus pallidus Externus (GPe), anterior internal capsule (ALIC), and nucleus accumbens (NA) are also considered useful in some studies [2]. It has been shown that stimulation of each target would lead to an improvement in specific set of components of the disease (e.g. tics, OCD and etc.) while it varies in different cases [2]. Anteromedial GPi is mostly known as an effective target for improvement of tic component of TS, while some studies have stated its ineffectiveness [2,3]. Stimulation of anteromedial GPi also has been shown to be an effective target in the treatment of the isolated OCD [4], whereas the studies with the purpose of TS treatment have mentioned no significant improvement in the OCD component of TS [2]. Consequently, there is no a consensus yet on how stimulation of anteromedial GPi affects different components of TS (either tics or OCD) although is it generally thought to be effective in TS treatment [3].

In the current case, fluctuations and variations in the therapeutic outcome of TS components have been observed, depended to the stimulation parameters. During sessions, the optimal setup was achieved based on alterations in symptoms and patient's compliance. With this setup, stimulation of anteromedial GPi led to significant improvement in OCD, but the tics were still existed and got worse progressively. To the best of our knowledge, stimulation of GPi with similar therapeutic outcome has not yet been reported.

In spite of many studies on how CSTC circuit may play an important role in Tic, OCD and TS, our observation along with many others are still needed to be explained in terms of variations in therapeutic outcomes. Many neural pathways are connecting different and specific parts of cerebral cortex, striatum and thalamus together, making multiple parallel and integrated circuits [5-7]. As an example, some areas in $\mathrm{GPi}$, projects neurons to specific parts of thalamus [5] and the same interconnections would exist between thalamus and cerebral cortex [7], GP and other part of striatum [5], GP and thalamus [5] and also in GP itself [5]. In this case, there exist multiple neural pathways between cerebral cortex, striatum and thalamus, involving specific part of each.

Among all these complicated circuits, some specific areas and pathways are related to either tic or OCD pathophysiology and some others are in common; while a portion of them remains unrelated to both. To be more detailed, anterior cingulate cortex, dorsal lateral prefrontal and lateral orbito-frontal areas are related to both OCD and tic disorders [1] which we intend to call them common areas; while, motor and oculomotor areas are mostly related to tic disorder [8] and ventromedial prefrontal cortex is mostly the characteristic of OCD [4]. Interestingly, common areas including lateral orbito-frontal area and anterior cingulate cortex are responsible for reward processing and fear expression, respectively, which are common features and underlying causes of both tics and OCD, whereas motor functions in tics may be subsequent to involvement of motor cortex and unwanted reoccurring thoughts in OCD may be due to the involvement of limbic system 
specifically $[1,4,8]$.

Anteromedial GPi is related to associative, motor and limbic areas through pallidothalamic and thalamocortical pathways [5-7, 9]. These pathways and areas may include the pathways or areas that are involved in the pathophysiology of either tic or OCD, or both. Therefore, Anteromedial GPi might be related to both common and tic-/OCD-specified areas and pathways and neural impulses from anteromedial GPi would find their way through specific neural pathways of pallidothalamic and thalamocortical pathways toward tic-/OCD-specified or common pathways and areas. DBS studies have also demonstrated that anteromedial GPi is a beneficial target for both tic and OCD [10] which indicates its involvement in both tic and OCD generating pathways and areas .

Here, we have hypothesized that through DBS procedure on anteromedial GPi, the stimulation impulse would travel through all possible related pathways to anteromedial GPi, including common or tic-/OCDspecified pathways and make an impact on any of them (figure 1). This impact may end up with an appropriate therapeutic outcome in either tic or OCD, or both in respect to physiological conditions of the pathways. In other words, physiological conditions of these pathways determine the variations in the therapeutic outcome. Moreover, it is probable that all tic or OCD generating circuits do not possess similar physiological conditions which leads to different responsiveness to certain stimulus. It can be said that specific stimulation adjustment may cause resynchronization as a probable effect in set of pathways, but causing no effect in the others in respect to their physiological condition. In our case, firstly we observed fluctuations in symptoms and eventually at the best adjustment, OCD improved significantly while tic remained unchanged. We think that stimulation of anteromedial GPi spread out to both tic-/OCD-specified pathways; and these pathways respond to the stimulus differently based on their physiological condition which led to fluctuations in symptoms. In other words, with certain adjustments, OCD-specified pathways probably resynchronized and improved better than tic-specified pathways in response to the stimulus because of the former's higher susceptibility to this event, leading to an improvement in OCD and not tic. In conclusion, we hypothesize that the anteromedial GPi can be described as a portal to the pathways involved in both tic and OCD and stimulation of this target would make an impact on all these pathways but with different extents. Hence, anteromedial GPi itself is not the determining factor in improvement of OCD or tic components of TS and the condition of involved pathways is playing an important role. This hypothesis would also explain variations in therapeutic outcomes of DBS for TS patients in other studies.

\section{Acknowledgement:}

We would like to acknowledge our patient whom cooperated with us kindly.

\section{Conflict of Interests:}

The authors of this article declare no conflict of interests.

Financial disclosure: None of the authors have received any funding of any kind for either this project or other projects.

Funding sources: No funding is received for this study.

Consent statement: Two different consent was obtained from patient for Both DBS surgery and reporting the clinical observation.

\section{Number of pages of the manuscript (including title page and references): 12 Pages}

\section{References:}

1. Robertson, M.M., et al., Gilles de la Tourette syndrome.Nature reviews Disease primers, 2017. 3 (1): p. $1-20$.

2. Casagrande, S.C., et al., Deep brain stimulation in Tourette's syndrome: evidence to date. Neuropsychiatric disease and treatment, 2019. $15:$ p. 1061. 
3. Martinez-Ramirez, D., et al., Efficacy and safety of deep brain stimulation in Tourette syndrome: the international Tourette syndrome deep brain stimulation public database and registry. JAMA neurology, 2018. 75 (3): p. 353-359.

4. Milad, M.R. and S.L. Rauch, Obsessive-compulsive disorder: beyond segregated cortico-striatal pathways. Trends in cognitive sciences, 2012. 16 (1): p. 43-51.

5. Gilani, I.A., et al., Assessing Topographic Structural Connectivity of the Human Basal Ganglia and Thalamus. bioRxiv, 2020.

6. Cacciola, A., et al., Structural connectivity-based topography of the human globus pallidus: Implications for therapeutic targeting in movement disorders. Movement Disorders, 2019. 34 (7): p. 987-996.

7. Yuan, R., et al., Functional topography of the thalamocortical system in human. Brain Structure and Function, 2016. 221 (4): p. 1971-1984.

8. Wang, Z., et al., The neural circuits that generate tics in Tourette's syndrome. American journal of psychiatry, 2011.168 (12): p. 1326-1337.

9. Bertino, S., et al., Spatially coherent and topographically organized pathways of the human globus pallidus. Human Brain Mapping, 2020.

10. Nair, G., et al., The anteromedial GPi as a new target for deep brain stimulation in obsessive compulsive disorder. Journal of Clinical Neuroscience, 2014. 21 (5): p. 815-821.

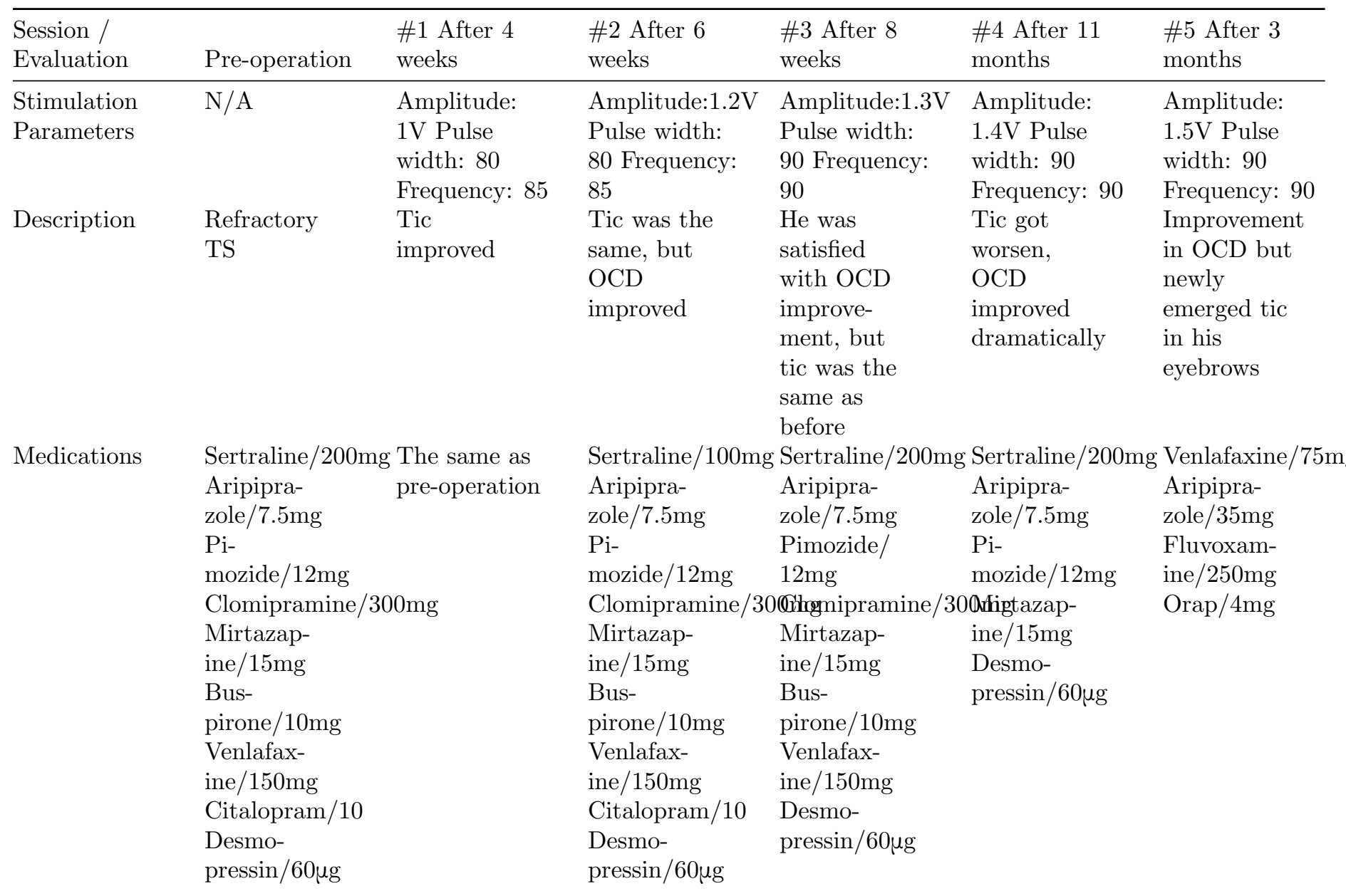




\begin{tabular}{lllllll}
\hline $\begin{array}{l}\text { Session / } \\
\text { Evaluation }\end{array}$ & Pre-operation & $\begin{array}{l}\text { \#1 After } 4 \\
\text { weeks }\end{array}$ & $\begin{array}{l}\text { \#2 After } 6 \\
\text { weeks }\end{array}$ & $\begin{array}{l}\text { \#3 After } 8 \\
\text { weeks }\end{array}$ & $\begin{array}{l}\text { \#4 After } 11 \\
\text { months }\end{array}$ & $\begin{array}{l}\text { \#5 After } 3 \\
\text { months }\end{array}$ \\
\hline Y-BOCS & 38 & 9 & 9 & 9 & 9 & 9 \\
PUTS & 28 & 23 & 23 & 23 & 23 & 23 \\
\hline
\end{tabular}

Table1: Pre-operation and follow up sessions data. Y-BOCS: Yale-Brown obsessive compulsive scale, PUTS: Premonitory Urge for Tics Scale
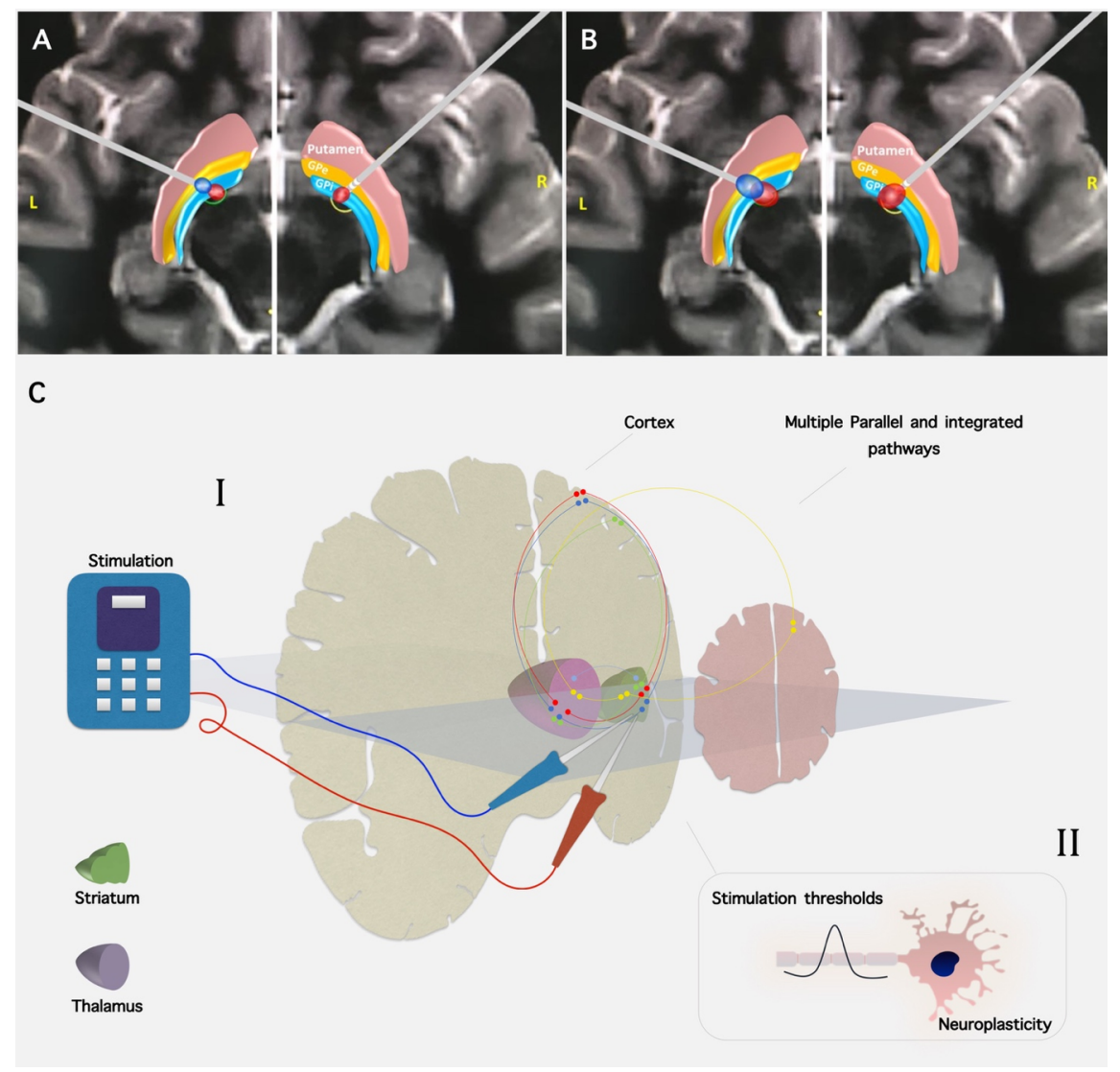

Figure1. Schematic illustration of the hypothesis. (A,B) Bilateral GPi lead location, globus pallidus internus (light blue), globus pallidus externus (orange), and putamen (pink), Volume of tissue activated (VTA) (red for negative and blue for positive) are shown for reference; (A) Volume of tissue activated (VTA) in the initial programming setting, L-GPi (3+;0-), R-GPi (C+;8-), 90 us pulse width, $90 \mathrm{~Hz}$ frequency, and 1.3 amplitude ; (B) VTA in the second programming setting: L-GPi L-GPi (3+;0-), R-GPi (C+;8-), 90 us pulse width, 90 $\mathrm{Hz}$ frequency, and 2.5 amplitude. (CI) Multiple parallel pathways connect different parts of cortex, Striatum and thalamus. Stimulation of a target (e.g. anteromedial GPi) as a member of these multiple parallel pathways, would spread through all different pathways related to anteromedial GPi and subsequently these pathways would response to the stimuli in a term of resynchronization as an embodiment. (CII) The response of can be varied in respect to pathways physiological condition including different stimulation thresholds and occurrence of neuroplasticity. 